\title{
Anatomy of enzyme channels
}

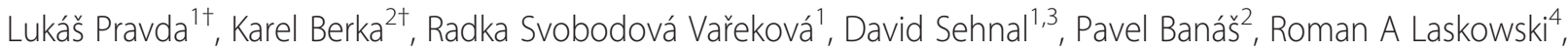 \\ Jaroslav Koča ${ }^{1^{*}}$ and Michal Otyepka ${ }^{2^{*}}$
}

\begin{abstract}
Background: Enzyme active sites can be connected to the exterior environment by one or more channels passing through the protein. Despite our current knowledge of enzyme structure and function, surprisingly little is known about how often channels are present or about any structural features such channels may have in common.

Results: Here, we analyze the long channels (i.e. >15 $\AA$ ) leading to the active sites of 4,306 enzyme structures. We find that over $64 \%$ of enzymes contain two or more long channels, their typical length being $28 \AA$. We show that amino acid compositions of the channel significantly differ both to the composition of the active site, surface and interior of the protein.

Conclusions: The majority of enzymes have buried active sites accessible via a network of access channels. This indicates that enzymes tend to have buried active sites, with channels controlling access to, and egress from, them, and that suggests channels may play a key role in helping determine enzyme substrate.
\end{abstract}

\section{Background}

Channels inside biomacromolecular structures (proteins, nucleic acids and their complexes) play many significant biological roles as they enable traffic between the interior spaces and the exterior. In enzymes they allow passage of substrates and products to/from the active site [1-12], in the ribosome they allow nascently synthetized proteins to pass from the proteosynthetic center to the outside [13], and in membrane proteins they provide high specificity of passage in either direction through the membrane $[14,15]$. Thus channels have attracted the attention of many researchers, who have rationalized their biological roles using a variety of experimental and theoretical methods. The ribosome, for example, prevents nascently synthetized polypeptides getting stuck in its polypeptide egress channel by lining the wall of the channel with a mosaic of alternating negative and positive electrostatic potentials $[13,16]$. Gramicidin provides polar holes for biomembranes, enabling free diffusion of

\footnotetext{
* Correspondence: jaroslav.koca@ceitec.muni.cz; michal.otyepka@upol.cz ${ }^{\dagger}$ Equal contributors

${ }^{1}$ National Centre for Biomolecular Research, Faculty of Science and CEITEC, Central European Institute of Technology, Masaryk University Brno, Kamenice 5, Brno-Bohunice 625 00, Czech Republic

${ }^{2}$ Department of Physical Chemistry, Regional Centre of Advanced Technologies and Materials, Faculty of Science, Palacký University Olomouc, tř. 17. listopadu 12, Olomouc 771 46, Czech Republic

Full list of author information is available at the end of the article
}

monovalent ions and water through the membrane [17-19], while transmembrane ion channels maintain their high selectivity by a combination of structural and electrostatic features of the channel-lining amino acids $[14,20]$.

Enzymes are proteins that catalyse reactions changing substrates to products. The enzymatic reactions occur in the enzymes' active sites. Thanks to the many analyses of enzymatic reactions, we now have a better understanding of how active site chemistry works [21-24] and which amino acids are present in the sites [25]. However, relatively little is known about how substrates enter active sites and how the respective products leave them. While some active sites are positioned on the protein's surface, in clefts or pockets, other enzymes have deeply buried active sites, which are connected to the outside by one or more channels. Here we focus on these channels, as the active site access paths play an important role in substrate and product trafficking between active site and outside. It has been shown that mutations in enzymes' active site access channels alter the substrate preferences of haloalkane dehalogenase enzymes and may be utilized in rational design of enzymes $[26,27]$. The amino acids lining the access channels of cytochrome P450 (CYP) are important for the selectivity of these enzymes [28] while the flexibility of these channels, i.e. their 
opening and closing motions, contributes to the broad substrate specificity of CYP $[10,29]$.

Despite a large effort, and recent progress in the field, an in-depth analysis of enzyme channels is lacking. Here, we use an advanced software tool, MOLE 2.0, developed for analysis of biomacromolecular channels [30], to survey 4,306 enzymes annotated in the Catalytic Site Atlas (CSA). We identify that more than $64 \%$ of enzyme structures contain channels at least $15 \AA$ long from the active site. A typical enzyme channel is $\sim 20 \AA$ long and its walls are made preferentially of histidine, arginine, tryptophan and tyrosine residues and, to a lesser extent, by phenylalanine, asparagine, and aspartic acid (Figure 1). These residues can be considered as gate-keepers controlling the entry to and from the active site.

\section{Results}

\section{Geometry features}

We identified that at least $64.2 \%$ of enzymes contain channels at least $15 \AA$ long (Table 1) and $86.8 \%$ contain channels at least $5 \AA$ long. However, as the short channels may correspond to paths connecting cleft-like active sites with the exterior, we decided to use only channels longer than $15 \AA$ for our analysis. Enzymes with channels (of $\geq 15 \AA$ in length) leading to a buried active site contained on average two such channels (Table 2). Some had more than five such channels, the highest number being 68 in 6,7dimethyl-8-ribityllumazine synthase from Aquifex aeolicus (PDBID: 1NQU; Additional file 1: Figure S1). Whereas one might expect larger proteins to contain larger numbers of channels, we found that the number of long channels does not correlate with protein size (Additional file 1:

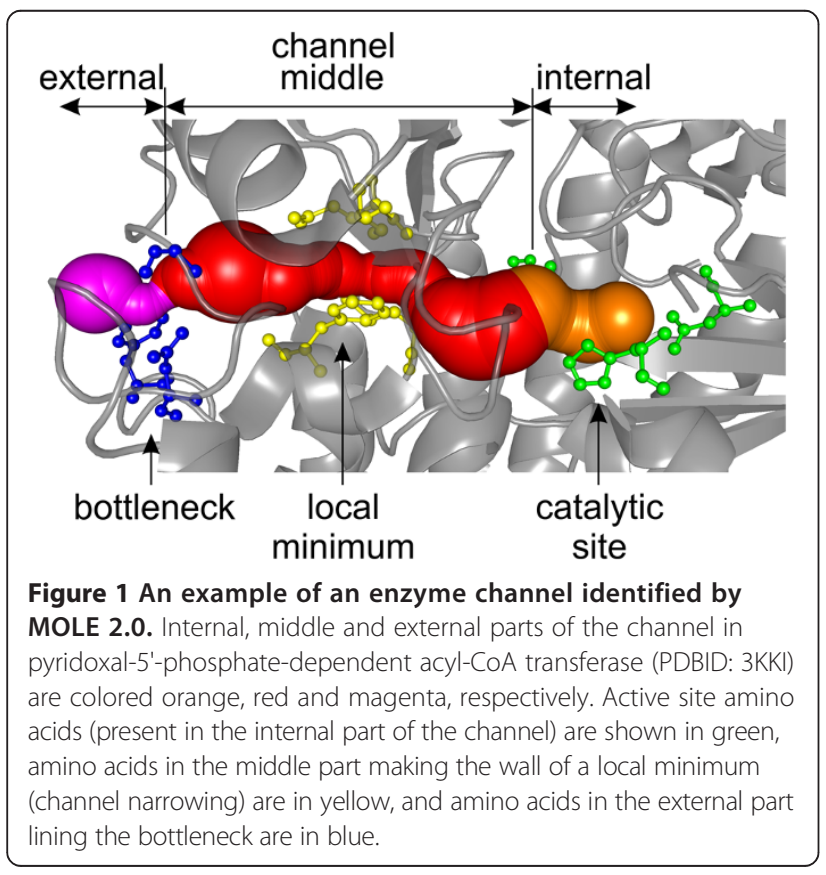

Table 1 Number of enzyme entries in each EC class, and numbers of channels of different lengths

\begin{tabular}{lllllll}
\hline EC & Enzyme class & $\begin{array}{l}\text { Number } \\
\text { of } \\
\text { enzymes }\end{array}$ & \multicolumn{3}{c}{ Enzymes with channels of length } \\
\cline { 3 - 7 } & & $\mathbf{2 5 \AA}$ & $\mathbf{2 1 0 ~ \AA}$ & $\mathbf{2 1 5} \AA$ & $\mathbf{2 0} \AA$ \\
\hline EC1 & Oxidoreductases & 879 & 781 & 736 & $\mathbf{6 8 4}$ & 612 \\
EC2 & Transferases & 1096 & 963 & 863 & $\mathbf{7 4 7}$ & 639 \\
EC3 & Hydrolases & 1455 & 1228 & 1019 & $\mathbf{7 4 9}$ & 542 \\
EC4 & Lyases & 465 & 401 & 361 & $\mathbf{3 1 8}$ & 262 \\
EC5 & Isomerases & 252 & 226 & 204 & $\mathbf{1 6 5}$ & 140 \\
EC6 & Ligases & 159 & 139 & 118 & $\mathbf{9 8}$ & 88 \\
& Sum & 4306 & 3738 & 3301 & $\mathbf{2 7 6 1}$ & 2283 \\
\hline
\end{tabular}

Numbers of enzymes in the dataset containing at least one channel of the given length. Bold values indicate $15 \AA$ threshold for channel detection used thorough the study.

Figure S2). The median channel length is $27.7 \AA$ (Table 2 and Additional file 1: Figure S3), 40\% of channels are 15$30 \AA$ long and $10 \%$ of enzymes contain channels longer than $50 \AA$. The longest channel (172 $\AA$ long) was found in Penicillium vitale catalase from Penicillium janthinellum (PDBID: 2IUF; Additional file 1: Figure S1). It should be noted that although the size of small enzymes (i.e. those containing fewer than 5,000 atoms) does not limit the number of long channels they contain, it does limit the maximum length these channels can have (Additional file 1: Figure S2).

Channel occurrence and length varies among the enzymatic classes (Table 2). The highest percentage (77.8\%) of proteins with channels longer than $15 \AA$ was identified in oxidoreductases (EC1), while the lowest percentage (51.8\%) applied to hydrolases (EC3). The number of channels is slightly elevated in oxidoreductases (EC1), transferases (EC2) and isomerases (EC5). Oxidoreductases (EC1) have median channel length longer by about $2 \AA$ than other enzymes, whereas transferases (EC2) and ligases (EC6) have average channel length shorter, also by about $2 \AA$ (Figure 2). As a result, oxidoreductases stand out of

Table 2 Geometrical channel features for all enzyme classes

\begin{tabular}{lllll}
\hline EC & $\mathbf{N a}$ & $\mathbf{P}$ & $\mathbf{M}^{\mathbf{a}}$ & $\mathbf{L}$ \\
\hline EC1 & 6518 & 77.8 & 3 & 29.8 \\
EC2 & 4728 & 68.2 & 3 & 26.3 \\
EC3 & 3454 & 51.8 & 2 & 27.5 \\
EC4 & 5780 & 68.4 & 2 & 27.9 \\
EC5 & 5107 & 65.5 & 3 & 26.4 \\
EC6 & 5289 & 61.6 & 2 & 25.2 \\
All & 4823 & 64.2 & 2 & 27.7
\end{tabular}

${ }^{a}$ Enzymes not containing channels were excluded.

Average number of atoms $(\mathrm{Na})$, percentage of enzymes containing at least one channel from the active site longer than $15 \AA$ ( $P$ in \%), median number of channels (M), and median length of channels ( $L$ in $\AA$ ) for each enzyme class. 


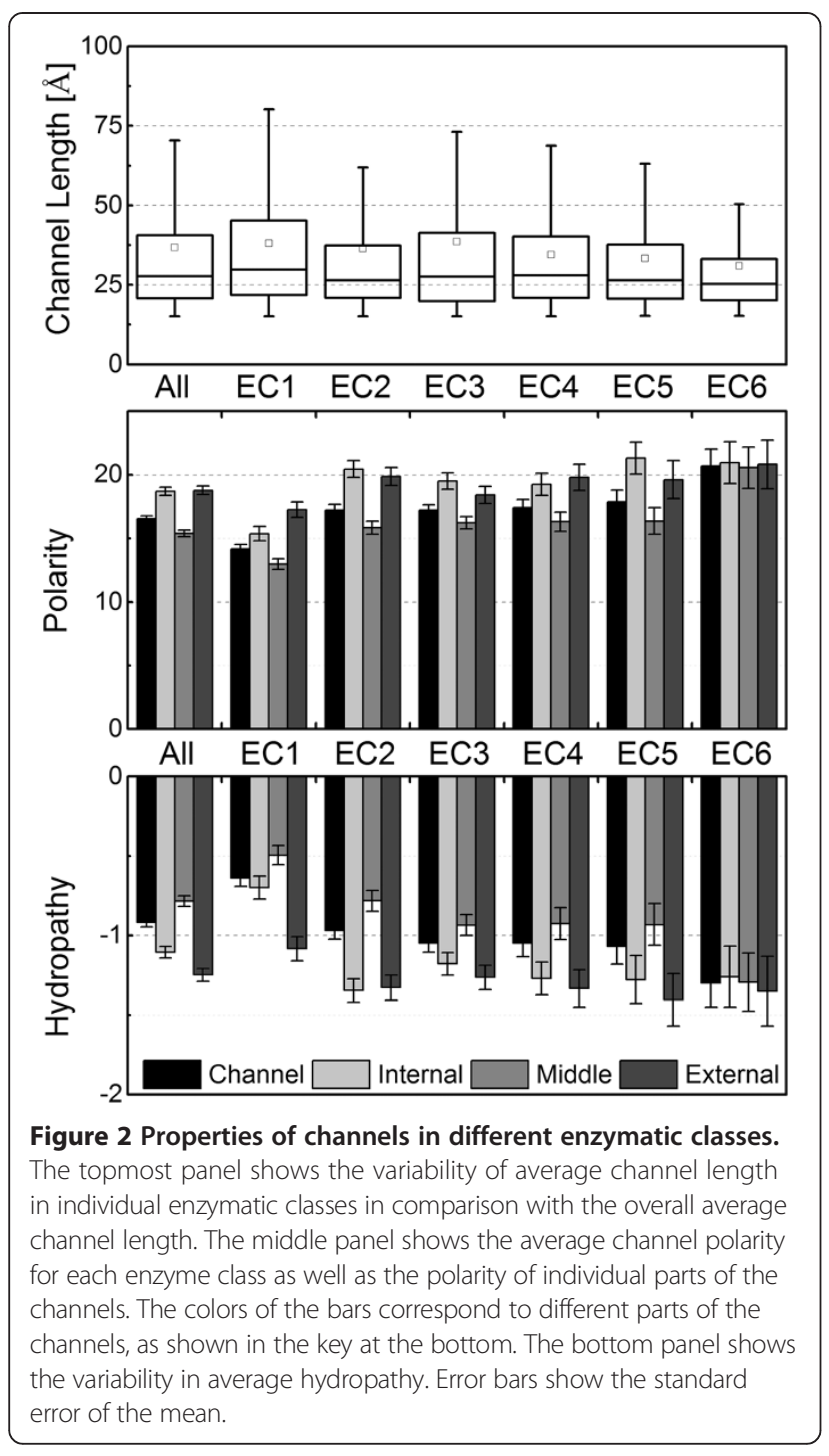

the crowd, as they have both higher channel occurrence and longer channels (Additional file 1: Figure S2).

\section{Physico-chemical features}

For each channel, we calculated basic physico-chemical features such as hydropathy [31] and polarity [32] using the method introduced in our previous paper [30]. In brief, the method sums up the length-weighted physicochemical properties of the amino acids lining the channel. As the algorithm is rather approximate, we would like to note that the estimated physico-chemical properties should be interpreted with care. The average channel hydropathy is -0.92 (Table 3) and this value is close to the hydropathy of tyrosine and tryptophan (it is worth noting that the hydropathy of amino acids varies from -4.5 of Arg to 4.5 of Ile). The distribution plots of hydropathy (Additional file 1: Figure S3) also indicate that the values are shifted in most channels to negative values indicating
Table 3 Physicochemical features of channels

\begin{tabular}{llllll}
\hline EC & Hydropathy & Polarity & $\mathbf{N}(+)$ & $\mathbf{N}(-)$ & $\boldsymbol{\Delta}$ \\
\hline EC1 & $-0.64 \pm 0.05$ & $14.2 \pm 0.4$ & 3 & 2 & 1 \\
EC2 & $-0.97 \pm 0.06$ & $17.2 \pm 0.4$ & 3 & 2 & 1 \\
EC3 & $-1.05 \pm 0.05$ & $17.2 \pm 0.4$ & 3 & 2 & 0 \\
EC4 & $-1.05 \pm 0.08$ & $17.4 \pm 0.6$ & 3 & 2 & 1 \\
EC5 & $-1.07 \pm 0.11$ & $17.9 \pm 0.9$ & 3 & 2 & 1 \\
EC6 & $-1.30 \pm 0.15$ & $20.7 \pm 1.3$ & 4 & 2 & 1 \\
All & $-\mathbf{0 . 9 2} \pm \mathbf{0 . 0 3}$ & $\mathbf{1 6 . 5} \pm \mathbf{0 . 2}$ & $\mathbf{3}$ & $\mathbf{2}$ & $\mathbf{0}$ \\
Internal & $-1.11 \pm 0.04$ & $18.7 \pm 0.3$ & 1 & 1 & 0 \\
Middle & $-0.78 \pm 0.03$ & $15.4 \pm 0.2$ & 1 & 0 & 0 \\
External & $-1.25 \pm 0.04$ & $18.8 \pm 0.3$ & 1 & 1 & 0 \\
\hline
\end{tabular}

The average hydropathy and polarity are shown for each enzyme class as well as for the internal, middle and external parts of channels. Also shown are the median number of charged amino acid side chains lining the channels $(\mathrm{N}(+)$ : positive Arg, Lys and His, $\mathrm{N}(-)$ : negative Asp and Glu, and $\Delta$ : median of overall charge). Bold values indicate features of all channels.

that hydrophilic channels are more preferred over hydrophobic ones.

The average channel polarity is 16.5 , which falls between the values of highly polar amino acids (Asp, Glu, Lys, Arg and His having polarities of $49.5-52.0$ ) and those of other amino acids (with polarities of $0.0-3.5$ ). It indicates that the channels are rather polar as well as hydrophilic. Taking all this information into account we may conclude that the average channel has slightly negative hydropathy and higher polarity. However, highly hydrophobic and nonpolar, as well as highly hydrophilic and polar, channels were also detected. We also analyzed the presence of charged amino acid side chains (Asp, Glu, His, Lys and Arg) in channels walls (Additional file 1: Table S2). On average the channel walls are lined by two negative and two positive side chains resulting in sum neutral channel walls (Table 3 ).

We also identified channels with significant extreme physico-chemical properties (Additional file 1: Table S3 and Additional file 1: Figure S1). Here we present two examples. A highly hydrophilic channel (hydropathy index -3.8) of length $18.7 \AA$ A occurs in 3-deoxy-D-arabinoheptulosonate-7-phosphate synthase (PDB ID: 1N8F) from E. coli. The high hydrophilicity of the channel is in accord with its function [33] since this enzyme catalyses a condensation reaction between two highly polar substrates: phosphoenolpyruvate and erythrose-4-phosphate. It is worth noting that transferases (EC2) show the largest variability in hydrophobicity as illustrated by the fact that six times transferases are in the top 10 having highly hydrophilic channels, and four occur in the top 10 with highly hydrophobic channels.

At the other extreme is peroxidase (PDBID: 1LYK) from the fungus Coprinus cinereus [34] which has a highly hydrophobic channel (hydropathy index of 3.59) 
of length $23.8 \AA$. This channel enables transport of simple phenols and smaller aromatic dye molecules for their oxidation in lignin decomposition [35] in a process which has been exploited in biotechnology as a dye-transfer inhibitor in a laundry detergent [36].

These examples show that enzymatic classes differ in their average physico-chemical properties: (i) oxidoreductases (EC1) show the most hydrophobic as well as the least polar channels among the enzyme classes, while (ii) ligases (EC6), and to some extent also isomerases (EC5), lyases (EC4) and hydrolases (EC3), show the most hydrophilic as well as the most polar channels (Figure 2, Table 3 and Additional file 1: Table S3).

We also identified that some physicochemical features differ across the three channel layers: internal, middle and external (Table 3). The polarity of middle part of the channel is always lower than polarity of both internal and external parts, respectively. The lower polarity of the middle part of channel is also reflected by its significantly more hydrophobic behaviour. The charged residues occur mainly in external parts of enzyme channels, while the internal and middle part contains more aromatic residues.

\section{Channel-lining residues}

We calculated the frequencies of channel-lining amino acids and compared them with frequencies of amino acids in the same enzyme structures. On the basis of this data, the channel propensities of individual amino acids can be defined as a ratio of the frequency of amino acid in the channel walls to the frequency of amino acid anywhere within the protein structure. The resulting channel propensities of the individual amino acids differ significantly. The rather bulky and aromatic amino acids (His, Tyr, Trp, Arg), occur over 1.25 times more frequently in the channel walls than in the whole enzyme. Additionally, other amino acids (Asn, Phe, Asp, Thr, Met, Ser) also show a slightly higher frequency in the channel walls than in the rest of the protein. Conversely, nonpolar aliphatic amino acids (Pro, Gly, Ile, Leu, Ala, and Val) are significantly less localized in channel walls (Figure 3). We also looked at the amino acid composition at each channel's local minimum. Whereas this reflected the composition of the whole channel, the channel bottlenecks contain significantly more cysteine (Cys), histidine (His) and tyrosine (Tyr) residues than usual and much fewer small aliphatic amino acids (Pro, Gly and Ala) (Additional file 1: Figure S4). As histidine (His) and cysteine (Cys) have unique binding properties, it is possible to hypothesize that these binding properties might provide a gate-keeping activity at the channel bottlenecks, whereas small aliphatic residues cannot undergo large changes and as such cannot serve as gate-keepers.

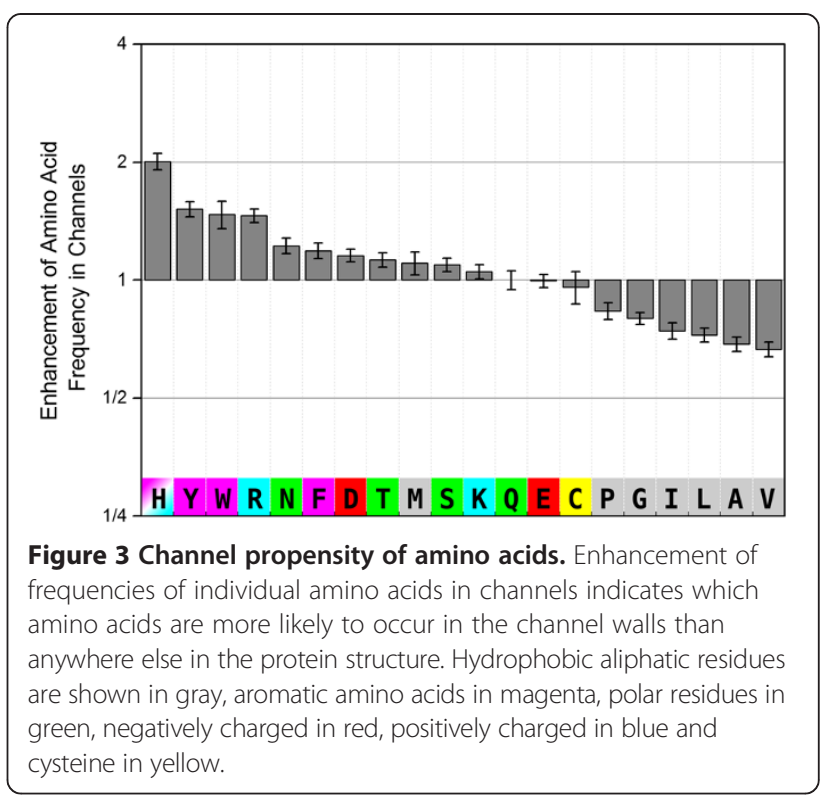

The frequencies of amino acids in active sites, on the protein surface and inside the protein, or in general channels, are markedly different from both the average protein amino acid composition and the composition of the channels (Figure 4). The active sites contain significantly more amino acids that can be part of a catalytic cycle (His, Asp, Cys, Glu, Arg, Tyr, Lys) enabling proton and electron shuffling and covalent bond reorganization. On the other hand, the frequency of less reactive amino acids (Trp, Thr, Gln, Phe) or amino acids with nonreactive side-chain (Met, Ala, Pro, Ile, Val, Leu) is lower in

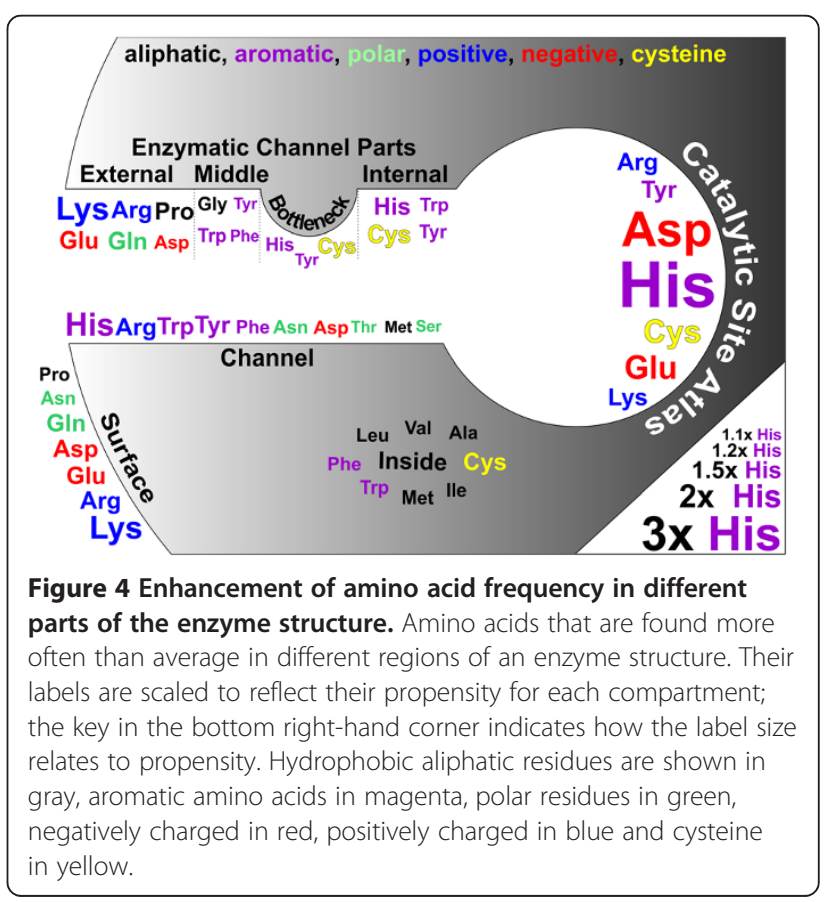


the active sites. These results are in perfect agreement with data published by Holliday and coworkers [37]. Protein cores are rich in hydrophobic aliphatic (Ile, Leu, Val, Ala, Met) and aromatic amino acids (Phe, Trp) as well as in cysteines (Cys). These amino acids have a structural function to maintain the formation and stability of protein hydrophobic cores [38] and the formation of disulphide bonds [39]. Conversely, the surface regions contain mainly charged (Lys, Arg, Glu, Asp) and polar residues (Asn, Gln), which facilitate contact with the polar water environment. Also, the surface has a higher than average frequency of prolines (Pro) as these helix-breaker amino acids are common in turns in the protein structures rigidifying the protein fold (Additional file 1: Figure S4).

Channel-lining amino acids are not uniformly distributed along the length of the channel. Internal parts of the channel tend to contain more aromatic residues (His, Trp, Tyr) together with cysteine (Cys), while glutamine and glutamate (Gln and Glu) and aliphatic amino acids (Pro, Ala, Leu) are underrepresented. These trends are similar to the catalytic site propensities, as discussed in the previous paragraph. The frequencies of amino acids in the middle regions of the channels correspond to the frequencies in the entire channel with the exception of glycine (Gly) and aromatic amino acids (Trp, Tyr, Phe), which are present more frequently. We may hypothesize that the higher frequency of glycine (Gly) in the middle channel parts is because it facilitates flexibility, which may be important for substrate/product channeling between the active site and protein surface [29], whereas aromatic amino acids can serve as gate-keepers. External parts of the channel bear more charged residues than any other part (Arg, Lys, Glu, Asp) together with proline (Pro) and glutamine (Gln), whereas other polar residues (Ser, Thr) are surprisingly less common in the external parts even though that both Ser and Thr are evenly distributed within the structure of proteins (Additional file 1: Figure S4). External parts of channels are less occupied by aliphatic (Ile, Ala, Val) and aromatic amino acids (Phe, Tyr) as well as typical catalytic amino acids - histidine (His), aspartate (Asp) and cysteine (Cys). A higher frequency of charged or rather bulky and aromatic amino acids in channels may have functional implications because such amino acids may work as gate-keepers, regulating traffic between active site and surface via conformational changes. It is worth noting that some mutations of such residues have been shown to alter the catalytic efficiency and substrate preferences in haloalkane dehalogenases [26].

All the results above concern channels leading to buried enzyme active sites. For comparison, we also analyzed all channels of length greater than $15 \AA$ connecting a cavity in the protein interior to the outside exterior (Additional file 1: Figure S4). We compared the amino acid compositions of the two types of channels. The active site access channels have higher frequencies of aromatic (Tyr, Trp and Phe), polar amino acids (Asn, Thr and Ser), catalytically active amino acids (Cys and His), and glycine (Gly). On the other hand, they contain fewer aliphatic amino acids (Pro, Leu, Ile and Val), charged and some polar amino acids (Lys, Glu, Arg and Gln). In sum, the active site access channels contain more functional amino acids than generic channels. This finding agrees with the idea that some amino acids bear functional roles in channels, e.g., as gate keepers or to maintain their flexibility.

The analysis of amino acids leading to active sites, divided according to the six enzymatic groups, shows that amino acid channel propensities correspond to overall channel propensities. However, some differences were identified (Additional file 1: Figure S4). As can be expected from their higher hydropathy and lower polarity, channels in oxidoreductases (EC1) have significantly lower frequencies of charged lining amino acids (Arg, Asp, Lys, Glu), but higher frequencies of aliphatic lining amino acids (Met, Pro, Ile, Leu, Ala, Val). Channels in transferases (EC2) contain fewer aromatic (Trp) and more charged (Arg, Asp, Lys) amino acids. Channels in hydrolases (EC3) contain fewer arginine (Arg), threonine (Thr) and aliphatic amino acids (Pro, Ile, Ala, Val), whereas they contain more aromatic (Trp, Tyr) and smaller charged (Lys, Asp, Glu) amino acids. Channels in lyases (EC4) show only lower amounts of aromatic (Trp) and sulphur containing (Met, Cys) amino acids. Channels in isomerases (EC5) contain fewer glycines (Gly). Channels in ligases (EC6) are the most hydrophilic channels, so it is not surprising that their channels contain fewer cysteine (Cys), aromatic (Trp, Tyr) and aliphatic (Pro, Leu) amino acids and more charged (Arg, Lys, Glu) amino acids and glycine (Gly). It should be noted that the differences between the individual enzyme classes should be interpreted with care because of larger statistical error bars, especially in the case of less populated EC5 and EC6 classes.

\section{Discussion}

Long channels (>15 $\AA$ ) are a common feature of enzymes, with over $64 \%$ containing at least two such channels. This shows that the majority of enzymes have buried active sites accessible via a network of access channels. Hence there is an apparent tendency for enzymes to bury their active site, i.e., to limit and control direct connection of active sites with the surrounding environment. This may be the result of two evolutionary pressures; i) steric, because active sites have to be structurally well arranged - a buried active site enables full spatial arrangement better than a pocket-like active site can give that half of the space of the latter is open to 
surrounding environment and ii) functional, as active site access paths may enable pre-selection of substrates, and may be involved in features co-determining enzyme substrate preferences. In another words, the active site access channels may limit access to the enzyme active sites and function as keyholes, enabling passage only of some classes of substrates.

The amino acid frequencies in the whole protein structures and channel walls differ significantly. Aliphatic amino acids are more involved in the formation of enzyme hydrophobic cores, which are important to maintain a protein fold. In turn, they are less frequently involved in channel wall lining or within the active sites. The aromatic, charged and polar amino acids occur more frequently in the channels walls. In addition, we identified a higher frequency of glycine in the middle parts of channels, which may function here to support channel flexibility enabling the channelling of bulkier substrates to active sites. This finding can be explained by the fact that the polar and charged amino acids line the channels to enable passage of polar substrates/products and water. The enhanced frequency of rather bulky and aromatic amino acids in channel external parts may have functional implications, because such amino acids may work as gatekeepers, regulating traffic between active site and outside.

The functional implications deduced from these global analyses are also supported by the fact that individual enzyme classes differ in their channel features. Typically oxidoreductases have the most hydrophobic, the least polar and longest channels among the enzyme classes, while ligases have the most hydrophilic, the most polar and the shortest channels. This indicates that evolution of enzymatic substrate preferences might also include evolution of active site access channels.

\section{Conclusions}

To conclude, we analyzed channels in 4,306 enzyme structures annotated in the Catalytic Site Atlas. We identified that at least $64 \%$ of enzyme structures contain on average two channels longer than $15 \AA$ leading to the catalytic site. Consequently, we may anticipate that the same number of enzymes have buried active sites. The longest, and also the most hydrophobic, channels are found in oxidoreductases, while the smallest number of channels can be found in hydrolases and the shortest and also the most hydrophilic channels in ligases. The composition of channel walls differs from the average composition of enzyme structures as well as from the composition of the protein surface. Hydrophobic aliphatic amino acids, which are the most common amino acids present in protein cores, occur in channel walls less frequently, whereas aromatic, charged and polar amino acids occur more frequently in channel walls. All these findings indicate that the active site access channels bear significant biological function as they are involved in co-determining enzyme substrate preferences.

\section{Methods}

Dataset

We analyzed 4,306 enzymes which were annotated in the Catalytic Site Atlas (CSA) database release of 4th March $2013[25,40]$. The dataset contained structures determined by X-ray diffraction at a resolution better than $2.5 \AA$, and had no two structures with a sequence identity higher than $90 \%$ (more quality checks can be found in Additional file 1: Table S1). It should be noted that when we used a dataset containing structures with a sequence identity less than $50 \%$, the results did not significantly differ from the results obtained with the dataset containing structures with a sequence identity less than $90 \%$. The enzymes in the dataset were grouped according to their Enzymatic Commission (EC) class (Table 1).

\section{Channel Identification}

An active site is a cavity, which walls contain amino acids residues annotated in the CSA. A channel is a pathway inside an active site cavity connecting the deepest apex of the cavity with an exterior. The MOLE 2.0 program [30] was used for channel identification and characterization. Briefly, the MOLE 2.0 algorithm calculates the Delaunay triangulation/Voronoi diagram of the atomic centers, splitting it into several smaller parts and identifying suitable start and end points in the interior and surface, respectively. Dijkstra's algorithm is used to identify tunnels as the shortest paths between the start and end points (see Additional file 1 for further details). This algorithm is used also in the MOLEonline 2.0 web application [41]. The setup of MOLE 2.0 for these calculations was as follows: Probe Radius and Origin Radius $5 \AA$, Interior Threshold $1.1 \AA$ and default values for Bottleneck Length, Bottleneck Radius, Cutoff Ratio and Surface Cover Radius. The CSA active sites were used as starting points. We used biological assemblies for the enzymes structures, which were obtained from the PDB database as *.pdb1 files. [42] Ten structures of EC 3.6.4 group were removed from the dataset. Hydrogen atoms and ligands not covalently bound to the structure were deleted prior to calculation. In cases where the system contained more than one active site, the site having the most channels was used. In order to study only relevant channels, we analysed only those channels longer than $15 \AA$. Table 1 shows the numbers of channels of different lengths for each of the six different enzyme classes, which are listed together with their properties in Additional file 2.

In the text we use the following terminology (Figure 1); Lining amino acids are all the amino acids fully encapsulating the detected channel and are divided into three 
classes: internal, middle, and external according to their respective positions in the layers perpendicular the channel centerline. Internal or external lining amino acids are those lying within a $5 \AA$ distance of the start or end point, respectively, with middle amino acids constituting the remainder. A bottleneck is where the channel radius is a minimum.

\section{Additional files}

Additional file 1: Description of MOLE methodology, and explanation of statistical methods used for channel analysis, Supplementary Figures S1-S4 and Supplementary Tables S1-S3.

Additional file 2: Full set of channels in csv format. The MOLE 2.0 application and enzymatic data set are available for download at http://mole.chemi.muni.cz/enzymes.

\section{Competing interests}

The authors declare that they have no competing interests.

\section{Authors' contributions}

LP carried out channel calculations. KB calculated the statistical analysis of the data and drafted the manuscript. RSV contributed to the design of the study and data interpretation. DS wrote the software used. PB participated in statistical analysis of the data. RAL participated in the design of the study and data interpretation. JK coordinated the study and wrote the manuscript. MO conceived of the study, designed and coordinated the study and wrote the manuscript. All authors read and approved the final manuscript.

\section{Acknowledgements}

This work was supported by the Czech Science Foundation [P208/12/G016 to M.O., P303/12/P019 to K.B.] and the Operational Program Research and Development for Innovations-European Regional Development Fund [CZ.1.05/2.1.00/03.0058 to M.O., K.B., P.B. and CZ.1.05/1.1.00/02.0068 to J.K. and R.S.V.], European Social Fund [CZ.1.07/2.3.00/20.0058 to K.B.]. D.S. acknowledges Brno City Municipality for financial support provided through the program Brno Ph.D. Talent. Access to the MetaCentrum supercomputing facilities provided under the research intent MSM6383917201 is gratefully acknowledged.

\section{Author details}

'National Centre for Biomolecular Research, Faculty of Science and CEITEC, Central European Institute of Technology, Masaryk University Brno, Kamenice 5, Brno-Bohunice 625 00, Czech Republic. ${ }^{2}$ Department of Physical Chemistry, Regional Centre of Advanced Technologies and Materials, Faculty of Science, Palacký University Olomouc, tř. 17. listopadu 12, Olomouc 771 46, Czech Republic. ${ }^{3}$ Faculty of Informatics, Masaryk University Brno, Botanická 68a, Brno 602 00, Czech Republic. ${ }^{4}$ European Molecular Biology Laboratory, European Bioinformatics Institute (EMBL-EBI), Wellcome Trust Genome Campus, Hinxton, Cambridge CB10 1SD, UK.

Received: 19 July 2014 Accepted: 5 November 2014 Published online: 18 November 2014

\section{References}

1. Huang X, Holden HM, Raushel FM: Channeling of substrates and intermediates in enzyme-catalyzed reactions. Annu Rev Biochem 2001, 70:149-180

2. Park J, Czapla L, Amaro RE: Molecular simulations of aromatase reveal new insights into the mechanism of ligand binding. J Chem Inf Model 2013, 53:2047-2056.

3. Sgrignani J, Magistrato A: Influence of the membrane lipophilic environment on the structure and on the substrate access/egress routes of the human aromatase enzyme. A computational study. J Chem Inf Model 2012, 52:1595-1606.
4. Madrona Y, Hollingsworth SA, Khan B, Poulos TL: P450cin active site water: implications for substrate binding and solvent accessibility. Biochemistry 2013, 52:5039-5050.

5. Cui Y-L, Zhang J-L, Zheng Q-C, Niu R-J, Xu Y, Zhang H-X, Sun C-C: Structural and dynamic basis of human cytochrome P450 7B1: a survey of substrate selectivity and major active site access channels. Chemistry 2013, 19:549-557.

6. Lee SJ, McCormick MS, Lippard SJ, Cho U-S: Control of substrate access to the active site in methane monooxygenase. Nature 2013, 494:380-384.

7. Pryor EE, Horanyi PS, Clark KM, Fedoriw N, Connelly SM, Koszelak-Rosenblum M, Zhu G, Malkowski MG, Wiener MC, Dumont ME: Structure of the integral membrane protein CAAX protease Ste24p. Science 2013, 339:1600-1604.

8. Xu S, Mueser TC, Marnett LJ, Funk MO: Crystal structure of 12-lipoxygenase catalytic-domain-inhibitor complex identifies a substrate-binding channel for catalysis. Structure 2012, 20:1490-1497.

9. Guskov A, Nordin N, Reynaud A, Engman H, Lundbäck A-K, Jong AJO, Cornvik T, Phua T, Eshaghi S: Structural insights into the mechanisms of Mg2+ uptake, transport, and gating by CorA. Proc Natl Acad Sci U S A 2012, 109:18459-18464.

10. Otyepka $M$, Berka $K$, Anzenbacher $P$ : Is there a relationship between the substrate preferences and structural flexibility of cytochromes P450? Curr Drug Metab 2012, 13:130-142.

11. Rengachari S, Aschauer P, Schittmayer M, Mayer N, Gruber K, Breinbauer R, Birner-Gruenberger R, Dreveny I, Oberer M: Conformational plasticity and ligand binding of bacterial monoacylglycerol lipase. J Biol Chem 2013, 288:31093-31104.

12. Salter MD, Blouin GC, Soman J, Singleton EW, Dewilde S, Moens L, Pesce A Nardini M, Bolognesi M, Olson JS: Determination of ligand pathways in globins: apolar tunnels versus polar gates. J Biol Chem 2012, 287:33163-33178.

13. Voss NR, Gerstein M, Steitz TA, Moore PB: The geometry of the ribosomal polypeptide exit tunnel. J Mol Biol 2006, 360:893-906.

14. Lemoine D, Jiang R, Taly A, Chataigneau T, Specht A, Grutter T: Ligand-gated Ion channels: new insights into neurological disorders and ligand recognition. Chem Rev 2012, 112:6285-6318.

15. Kasianowicz JJ: Introduction to Ion channels and disease. Chem Rev 2012 112:6215-6217.

16. Knight AM, Culviner PH, Kurt-Yilmaz N, Zou T, Ozkan SB, Cavagnero S: Electrostatic effect of the ribosomal surface on nascent polypeptide dynamics. ACS Chem Biol 2013, 8:1195-1204.

17. Eisenberg B: lonic channels in biological membranes: natural nanotubes. Acc Chem Res 1998, 4842:117-123.

18. Wallace B: Gramicidin channels and pores. Annu Rev Biophys Biophys Chem 1990, 19:127-157.

19. Roux B: Computational studies of the gramicidin channel. Acc Chem Res 2002, 35:366-375.

20. Maffeo C, Bhattacharya S, Yoo J, Wells D, Aksimentiev A: Modeling and simulation of lon channels. Chem Rev 2012, 112:6250-6284.

21. Kraut DA, Carroll KS, Herschlag D: Challenges in enzyme mechanism and energetics. Annu Rev Biochem 2003, 72:517-571.

22. Warshel A, Sharma PK, Kato M, Xiang Y, Liu H, Olsson MHM: Electrostatic basis for enzyme catalysis. Chem Rev 2006, 106:3210-3235.

23. Garcia-Viloca M, Gao J, Karplus M, Truhlar DG: How enzymes work: analysis by modern rate theory and computer simulations. Science 2004, 303:186-195.

24. Benkovic S, Hammes-Schiffer S: A perspective on enzyme catalysis. Science 2003, 301:1196-1202.

25. Porter CT, Bartlett GJ, Thornton JM: The catalytic site atlas: a resource of catalytic sites and residues identified in enzymes using structural data. Nucleic Acids Res 2004, 32(Database issue):D129-D133.

26. Pavlova M, Klvana M, Prokop Z, Chaloupkova R, Banas P, Otyepka M, Wade RC, Tsuda M, Nagata Y, Damborsky J: Redesigning dehalogenase access tunnels as a strategy for degrading an anthropogenic substrate. Nat Chem Biol 2009, 5:727-733.

27. Stepankova V, Khabiri M, Brezovsky J, Pavelka A, Sykora J, Amaro M, Minofar B, Prokop Z, Hof M, Ettrich R, Chaloupkova R, Damborsky J: Expansion of access tunnels and active-site cavities influence activity of haloalkane dehalogenases in organic cosolvents. Chembiochem 2013, 14:890-897.

28. Skopalík J, Anzenbacher P, Otyepka M: Flexibility of human cytochromes P450: molecular dynamics reveals differences between CYPs 3A4, 2C9, and $2 \mathrm{A6}$, which correlate with their substrate preferences. J Phys Chem $B$ 2008, 112:8165-8173. 
29. Hendrychová T, Berka K, Navrátilová V, Anzenbacher P, Otyepka M: Dynamics and hydration of the active sites of mammalian cytochromes $\mathrm{P} 450$ probed by molecular dynamics simulations. Curr Drug Metab 2012, 13:177-189.

30. Sehnal D, Svobodová Vařeková R, Berka K, Pravda L, Navrátilová V, Banáš P, Ionescu C-M, Otyepka M, Koča J: MOLE 2.0: advanced approach for analysis of biomacromolecular channels. J Cheminform 2013, 5:39.

31. Kyte J, Doolittle RF: A simple method for displaying the hydropathic character of a protein. J Mol Biol 1982, 157:105-132.

32. Zimmerman $\mathrm{JM}$, Eliezer $\mathrm{N}$, Simha R: The characterization of amino acid sequences in proteins by statistical methods. J Theor Biol 1968, 21:170-201.

33. Webby CJ, Lott JS, Baker HM, Baker EN, Parker E: Crystallization and preliminary X-ray crystallographic analysis of 3-deoxy-D-arabino-heptulosonate-7phosphate synthase from Mycobacterium tuberculosis. Acta Crystallogr Sect F: Struct Biol Cryst Commun 2005, 61(Pt 4):403-406.

34. Houborg K, Harris P, Petersen J, Rowland P, Poulsen J-CN, Schneider P, Vind J, Larsen S: Impact of the physical and chemical environment on the molecular structure of Coprinus cinereus peroxidase. Acta Crystallogr Sect D: Biol Crystallogr 2003, D59:989-996.

35. Lundell TK, Mäkelä MR, Hildén K: Lignin-modifying enzymes in filamentous basidiomycetes-ecological, functional and phylogenetic review. J Basic Microbiol 2010, 50:5-20.

36. Cherry JR, Lamsa MH, Schneider P, Vind J, Svendsen A, Jones A, Pedersen AH: Directed evolution of a fungal peroxidase. Nat Biotechnol 1999, 17:379-384.

37. Holliday GL, Mitchell JBO, Thornton JM: Understanding the functional roles of amino acid residues in enzyme catalysis. J Mol Biol 2009, 390:560-577.

38. Dill KA: Dominant forces in protein folding. Biochemistry 1990, 29:7133-7155.

39. Wilkinson B, Gilbert HF: Protein disulfide isomerase. Biochim Biophys Acta 2004, 1699:35-44.

40. Furnham N, Holliday GL, de Beer TAP, Jacobsen JOB, Pearson WR, Thornton JM: The catalytic site atlas 2.0: cataloging catalytic sites and residues identified in enzymes. Nucleic Acids Res 2014, 42:D485-D489.

41. Berka K, Hanák O, Sehnal D, Banáš P, Navrátilová V, Jaiswal D, Ionescu C-M, Svobodová Vařeková R, Koča J, Otyepka M: MOLEonline 2.0: interactive web-based analysis of biomacromolecular channels. Nucleic Acids Res 2012, 40(Web Server issue):W222-W227.

42. Berman $\mathrm{H}$, Henrick K, Nakamura H, Markley JL: The worldwide Protein Data Bank (wwPDB): ensuring a single, uniform archive of PDB data. Nucleic Acids Res 2007, 35(Database issue):D301-D303.

doi:10.1186/s12859-014-0379-x

Cite this article as: Pravda et al:: Anatomy of enzyme channels. BMC Bioinformatics 2014 15:379.

\section{Submit your next manuscript to BioMed Central and take full advantage of:}

- Convenient online submission

- Thorough peer review

- No space constraints or color figure charges

- Immediate publication on acceptance

- Inclusion in PubMed, CAS, Scopus and Google Scholar

- Research which is freely available for redistribution 